\title{
Canonical Quantization of the Scalar Field: The Measure Theoretic Perspective
}

\author{
José Velhinho \\ Faculdade de Ciências, Universidade da Beira Interior, R. Marquês D’Ávila e Bolama, 6201-001 Covilhã, Portugal \\ Correspondence should be addressed to José Velhinho; jvelhi@ubi.pt
}

Received 16 August 2015; Accepted 2 September 2015

Academic Editor: Ivan Avramidi

Copyright (C) 2015 José Velhinho. This is an open access article distributed under the Creative Commons Attribution License, which permits unrestricted use, distribution, and reproduction in any medium, provided the original work is properly cited.

\begin{abstract}
This review is devoted to measure theoretical methods in the canonical quantization of scalar field theories. We present in some detail the canonical quantization of the free scalar field. We study the measures associated with the free fields and present two characterizations of the support of these measures. The first characterization concerns local properties of the quantum fields, whereas for the second one we introduce a sequence of variables that test the field behaviour at large distances, thus allowing distinguishing between the typical quantum fields associated with different values of the mass.
\end{abstract}

\section{Introduction}

The phase space of the classical scalar field is a linear space and therefore constitutes an infinite dimensional analogue of the usual phase space for classical dynamics of a finite number of particles, namely, the cotangent bundle $T^{*} \mathbb{R}^{n}$. The usual finite dimensional Heisenberg kinematical algebra admits a natural generalization in this infinite dimensional context: the kinematical variables are conveniently labelled by smooth test functions, belonging to the real Schwartz space $\delta\left(\mathbb{R}^{d}\right)$. The Heisenberg group and the Weyl relations admit suitable generalizations as well, and therefore the problem of the canonical quantization of kinematical observables in scalar field theory is, a priori, well defined: following Weyl, Gelfand, and Segal, one should look for representations of the Weyl relations $[1,2]$. However, in contrast to what happens in finite dimensions, the Weyl relations in field theory admit nonequivalent representations; that is, the quantization of the kinematics is not unique. Note that this is definitely not only due to the existence of pathological representations: examples of physically relevant nonequivalent representations are those associated with free fields with different masses [2]. Moreover, the quantization of the kinematics of theories with interactions is not equivalent to the quantization of the kinematics of free theories $[3,4]$. So, the dynamics plays a crucial role in the selection/construction of a quantum representation adequate to a given classical field model already at the kinematical level. The issue of the unitary representation, at the quantum level, of natural symmetries of the classical model, for example, the Poincaré group, is also important. Note that a unitary representation of the Poincaré group includes a quantization of the dynamics, given by the representation of the subgroup of time translations. This is essentially the problem of quantization of field theories: given a certain classical model, the issue is to construct a quantum representation with the appropriate invariance properties, therefore allowing a consistent quantization of the dynamics and of relevant symmetry groups.

The theory of representations of the Weyl relations can be seen as a problem in measure theory in infinite dimensional linear spaces. Just as in finite dimensions, it would be natural to look for representations in spaces of square integrable functions with respect to some measure on the classical configuration space, which is a space of functions in $\mathbb{R}^{d}$. It turns out, however, that potentially interesting measures on those spaces fail to satisfy the crucial property of $\sigma$ additivity, which, in particular, implies that one cannot obtain Hilbert spaces out of the measures. To obtain $L^{2}$ spaces it is necessary to extend the classical configuration space to spaces of distributions. Spaces of distributions are indeed 
the natural "home" to interesting measures in field theory. In order to obtain representations of the Weyl relations it is sufficient to consider the space of distributions $\mathcal{S}^{\prime}\left(\mathbb{R}^{d}\right)$, dual of the Schwartz space of test functions $\delta\left(\mathbb{R}^{d}\right)$. In fact, one can show that there is a one-to-one correspondence between (cyclic) representations and certain classes of measures in $\delta^{\prime}\left(\mathbb{R}^{d}\right)$ [5]. These measures have the property of being quasiinvariant with respect to the action of $\mathcal{S}\left(\mathbb{R}^{d}\right)$ as translations in $\mathcal{S}^{\prime}\left(\mathbb{R}^{d}\right)$, which essentially means that the translation of such a measure by an element of $\delta\left(\mathbb{R}^{d}\right)$ produces a measure supported on the same subset of $\mathcal{S}^{\prime}\left(\mathbb{R}^{d}\right)$. Measures $\mu$ of this type define representations of the Weyl relations in the Hilbert space of square integrable functions $L^{2}\left(\mathcal{S}^{\prime}\left(\mathbb{R}^{d}\right), \mu\right)$ [5]. The distribution space $\mathcal{S}^{\prime}\left(\mathbb{R}^{d}\right)$ can therefore be seen as the "universal quantum configuration space" for the real scalar field.

In the present review we discuss in detail the canonical quantization of the free massive scalar field, following [ 1 , $2,5,6]$, including also an analysis of the support of the corresponding measures. This latter study follows closely $[7,8]$, which in turn were partly inspired by [9-11]. In Section 2 we have collected a minimal amount of relevant notions and results concerning Gaussian representations of the Weyl relations. Section 3 deals with the classical dynamics in the Hamiltonian formalism: since the equations of motion are linear, the solution is given by a one-parameter group of linear symplectic transformations. In Section 4 we discuss the unitary implementation in the quantum theory of linear symplectomorphisms in the context of Gaussian representations of the Weyl relations. In Section 5 we present the Gaussian measure corresponding to the free quantum field. We will see that for each value of the mass there is a corresponding Gaussian measure allowing a physically consistent quantization of the dynamics. Quantum dynamics is given by a one-parameter group of unitary transformations in perfect correspondence with the classical situation. One can show that the quantum Hamiltonian is a positive operator and that there is a unique vacuum. Moreover, it can be shown that these conditions determine a unique quantum representation [1]. In Section 6 we show that the free field measure is invariant and ergodic with respect to the action of the Euclidean group on $\mathbb{R}^{d}$. This result gives us a unitary representation of the Euclidean group on the quantum Hilbert space and shows that the vacuum is the only invariant state. Since this is true for every value of the mass, it also shows that the representations of the Weyl relations corresponding to two different values of the mass are not unitarily equivalent. In Section 7 we discuss briefly the relativistic invariance properties of the free field quantization. The last two sections concern properties of the support of the free field measures. In Section 8 local properties of the support are discussed. In Section 9 we analyse the long range behaviour instead, which will allow us to distinguish between the supports of the measures associated with different values of the mass.

\section{Gaussian Representations of the Weyl Relations}

The canonical quantization of field theories involves the introduction of a convenient measure in an infinite dimensional space. In the case of the real scalar field the appropriate measure space is the dual of the Schwartz space. Measures in this space allow the construction of representations of the Weyl relations which are of the Schrödinger type. In particular, the measure associated with the free field is a Gaussian measure.

2.1. Gaussian Measures on $\mathcal{S}^{\prime}\left(\mathbb{R}^{d}\right)$. Let $\mathcal{S}^{\prime}\left(\mathbb{R}^{d}\right)$ be the topological dual of the real Schwartz space $\delta\left(\mathbb{R}^{d}\right)$ with respect to the nuclear topology. We will consider $\mathcal{S}^{\prime}\left(\mathbb{R}^{d}\right)$ as a measurable space, with the $\sigma$-algebra of measurable sets being the smallest $\sigma$-algebra such that all the maps, $\phi \mapsto \phi(f)$, $\phi \in \mathcal{S}^{\prime}\left(\mathbb{R}^{d}\right)$, and $f \in \mathcal{S}\left(\mathbb{R}^{d}\right)$, are measurable. This $\sigma$-algebra coincides with the Borel $\sigma$-algebra associated with the strong topology on $\mathcal{S}^{\prime}\left(\mathbb{R}^{d}\right)$.

Definition 1. The Fourier transform of a measure $\mu$ on $\mathcal{S}^{\prime}\left(\mathbb{R}^{d}\right)$ is the function $\chi: S\left(\mathbb{R}^{d}\right) \rightarrow \mathbb{C}$ defined by

$$
\chi(f)=\int_{\mathcal{S}^{\prime}\left(\mathbb{R}^{d}\right)} e^{i \phi(f)} d \mu(\phi) .
$$

Definition 2. A complex function $\chi$ on a linear space $E$ is said to be of positive type if

$$
\begin{aligned}
\sum_{k, l=1}^{m} c_{k} \bar{c}_{l} \chi\left(\xi_{k}-\xi_{l}\right) \geq 0 & \\
\forall m & \in \mathbb{N}, c_{1}, \ldots, c_{m} \in \mathbb{C}, \xi_{1}, \ldots, \xi_{m} \in E .
\end{aligned}
$$

Theorem 3 (Bochner-Minlos). The Fourier transform of a measure on $\mathcal{S}^{\prime}\left(\mathbb{R}^{d}\right)$ is a positive type function, continuous with respect to the nuclear topology. Conversely, a continuous function of positive type on $\mathcal{S}\left(\mathbb{R}^{d}\right)$ is the Fourier transform of a uniquely defined measure on $\mathcal{S}^{\prime}\left(\mathbb{R}^{d}\right)$.

Definition 4. Let (, ) be a continuous inner product on $\mathcal{S}\left(\mathbb{R}^{d}\right)$. The Gaussian measure on $\mathcal{S}^{\prime}\left(\mathbb{R}^{d}\right)$ of covariance $($,$) is the$ measure whose Fourier transform is $\chi(f)=e^{-(f, f) / 2}, f \in$ $\mathcal{S}\left(\mathbb{R}^{d}\right)$.

Note that, given a Gaussian measure $\mu$ of covariance (, ), every element of the real Hilbert space $\mathscr{H}$, the completion of $\mathcal{S}\left(\mathbb{R}^{d}\right)$ with respect to $($,$) , still defines an element of$ $L^{1}\left(\mathcal{S}^{\prime}\left(\mathbb{R}^{d}\right), \mu\right)$, generalizing $\phi \mapsto \phi(f)$. This follows (see, e.g., [12]) from the obvious fact that the Fourier transform is continuous with respect to (, ) norm.

We are particularly interested in the case where the covariance is defined by certain types of linear operators on $\mathcal{S}\left(\mathbb{R}^{d}\right)$.

Definition 5. We will say that a linear operator $C: \mathcal{S}\left(\mathbb{R}^{d}\right) \rightarrow$ $\delta\left(\mathbb{R}^{d}\right)$ is a covariance operator if

(i) $C$ is a homeomorphism of $\mathcal{S}\left(\mathbb{R}^{d}\right)$ with respect to the nuclear topology; 
(ii) $C$ is bounded, self-adjoint, and positive on $L^{2}\left(\mathbb{R}^{d}\right)$;

(iii) $C^{-1}$, seen as a densely defined operator on $L^{2}\left(\mathbb{R}^{d}\right)$, is (essentially) self-adjoint and positive.

It is clear that the bilinear form

$$
\langle f, g\rangle_{C}:=\langle f, C g\rangle, \quad f, g \in \mathcal{S}\left(\mathbb{R}^{d}\right)
$$

defines an inner product, for any covariance operator, where $\langle$,$\rangle denotes L^{2}\left(\mathbb{R}^{d}\right)$ inner product; that is,

$$
\langle f, g\rangle:=\int_{\mathbb{R}^{d}} f g d^{d} x .
$$

A covariance operator $C$ defines thus a Gaussian measure, and we will say also that $C$ is the covariance of the measure.

2.2. Representations of the Weyl Relations. In the canonical quantization of real scalar field theories in $d+1$ dimensions one looks for unitary representations of the Weyl relations. Consider

$$
\mathscr{V}(g) \mathcal{U}(f)=e^{i\langle f, g\rangle} \mathcal{U}(f) \mathscr{V}(g),
$$

where $f$ and $g$ belong to $\mathcal{S}\left(\mathbb{R}^{d}\right)$. By representation of the above relations a pair $(\mathscr{U}, \mathscr{V})$ of strongly continuous unitary representations (on the same Hilbert space) of the commutative nuclear group $\delta\left(\mathbb{R}^{d}\right)$ is meant. It is also required that the combined action of $\mathscr{U}$ and $\mathscr{V}$ be irreducible. A given representation on a Hilbert space $\mathscr{H}$ is said to be cyclic if there is $\theta \in \mathscr{H}$ such that the linear space of $\left\{\mathscr{U}(f) \theta, f \in \mathcal{S}\left(\mathbb{R}^{d}\right)\right\}$ is dense in $\mathscr{H}$. We will consider only cyclic representations.

It is a well established fact [5] that cyclic representations are in one-to-one correspondence with quasi-invariant measures on $\mathcal{S}^{\prime}\left(\mathbb{R}^{d}\right)$. Consider first the action of $\mathcal{S}\left(\mathbb{R}^{d}\right)$ as translations of $\mathcal{S}^{\prime}\left(\mathbb{R}^{d}\right)$ :

$$
\mathcal{S}^{\prime}\left(\mathbb{R}^{d}\right) \ni \phi \longmapsto \phi+f, \quad f \in \mathcal{S}\left(\mathbb{R}^{d}\right),
$$

where $\phi+f$ is defined by $(\phi+f)(g)=\phi(g)+\langle f, g\rangle$, $\forall g \in \mathcal{S}\left(\mathbb{R}^{d}\right)$. A measure $\mu$ on $\mathcal{S}^{\prime}\left(\mathbb{R}^{d}\right)$ is quasi-invariant (with respect to the above action) if the translated measures $\mu_{f}(\phi):=\mu(\phi-f)$ are mutually absolutely continuous with respect to $\mu, \forall f \in \mathcal{S}\left(\mathbb{R}^{d}\right)$. (Note that there are no quasiinvariant measures with respect to all the translations on $\mathcal{S}^{\prime}\left(\mathbb{R}^{d}\right)$. This fact is ultimately responsible for the existence of nonequivalent representations of the Weyl relations, in contrast with the corresponding situation in finite dimensions.)

Given a quasi-invariant measure $\mu$, one defines a cyclic representation of the Weyl relations on $L^{2}\left(\mathcal{S}^{\prime}\left(\mathbb{R}^{d}\right), \mu\right)$ by the following actions of $\mathscr{U}$ and $\mathscr{V}$ :

$$
\begin{aligned}
& (\mathscr{U}(f) \psi)(\phi)=e^{-i \phi(f)} \psi(\phi), \\
& (\mathscr{V}(g) \psi)(\phi)=\left(\frac{d \mu(\phi-g)}{d \mu(\phi)}\right)^{1 / 2} \psi(\phi-g) .
\end{aligned}
$$

The representation $\mathscr{V}$ is unitary precisely because the measure is quasi-invariant, allowing the existence of the RadonNikodym derivative.
The following proposition gives necessary and sufficient conditions for the equivalence of cyclic representations. We introduce the Weyl operators $\mathscr{W}(f, g):=e^{i\langle f, g\rangle / 2} \mathscr{U}(f) \mathscr{V}(g)$.

Proposition 6. Two cyclic representations $(\mathscr{H}, \mathscr{W})$ and $\left(\mathscr{H}^{\prime}, \mathscr{W}^{\prime}\right)$ with cyclic vectors $\theta$ and $\theta^{\prime}$, respectively, satisfy

$$
\left\langle\theta^{\prime}, \mathscr{W}^{\prime}(f, g) \theta^{\prime}\right\rangle=\langle\theta, \mathscr{W}(f, g) \theta\rangle, \quad \forall f, g
$$

if and only if there is a unitary operator $T: \mathscr{H}^{\prime} \rightarrow \mathscr{H}$ such that $T \mathscr{W}^{\prime}(f, g) T^{-1}=\mathscr{W}(f, g)$ and $T \theta^{\prime}=\theta$.

Representations (7) and (8) are irreducible if and only if the measure is ergodic with respect to the action (6) of $\mathcal{S}\left(\mathbb{R}^{d}\right)$ [1]. Moreover, one can show that two ergodic measures give rise to unitarily equivalent representations if and only if the two measures are mutually absolutely continuous. Well known examples of quasi-invariant and ergodic measures on $\mathcal{S}^{\prime}\left(\mathbb{R}^{d}\right)$ are provided by Gaussian measures. We will say that the corresponding representations of the Weyl relations are Gaussian representations.

Let then $C$ be a covariance operator on $\delta\left(\mathbb{R}^{d}\right)$ and let $\mu$ be the corresponding measure on $\mathcal{S}^{\prime}\left(\mathbb{R}^{d}\right)$. The RadonNikodym derivative in (8) is easy to evaluate and one thus has the following irreducible representation of the Weyl relations defined by the covariance $C$ :

$$
\begin{aligned}
& (\mathscr{U}(f) \psi)(\phi)=e^{-i \phi(f)} \psi(\phi), \\
& (\mathscr{V}(g) \psi)(\phi)=e^{-\left\langle g, C^{-1} g\right\rangle / 4} e^{\phi\left(C^{-1} g\right) / 2} \psi(\phi-g) .
\end{aligned}
$$

One can easily evaluate the expectation values of the Weyl operators on the cyclic vector:

$$
\langle 1, \mathscr{W}(f, g) 1\rangle=e^{-\langle\langle(f, g),(f, g)\rangle\rangle_{C} / 4},
$$

where $\langle\langle,\rangle\rangle_{C}$ is the inner product on $\mathcal{S}\left(\mathbb{R}^{d}\right) \oplus \mathcal{S}\left(\mathbb{R}^{d}\right)$ :

$$
\begin{aligned}
& \left\langle\left\langle(f, g),\left(f^{\prime}, g^{\prime}\right)\right\rangle\right\rangle_{C} \\
& \quad:=\langle f,(2 C) f\rangle+\left\langle g,(2 C)^{-1} g\right\rangle .
\end{aligned}
$$

In the case of Gaussian representations the natural topology on the test functions space is determined by the inner product $\langle\langle,\rangle\rangle_{C}$. It is not difficult to show that $\mathscr{U}(10)$ and $\mathscr{V}(11)$ are in fact continuous with respect to the inner products $\langle\cdot, C \cdot\rangle$ and $\left\langle\cdot, C^{-1} \cdot\right\rangle$, respectively. Therefore, the Weyl operators are well defined for all $f, g \in \mathscr{H}_{C} \oplus \mathscr{H}_{\mathrm{C}^{-1}}$, the real completion of $\mathcal{S}\left(\mathbb{R}^{d}\right) \oplus \mathcal{S}\left(\mathbb{R}^{d}\right)$ with respect to $\langle\langle,\rangle\rangle_{C}$. Equation (12) for the expectation values still hold.

\section{Classical Free Field Dynamics}

Let us consider the free scalar field of mass $m$ in $d+1$ dimensions whose dynamics is given by the Klein-Gordon equation:

$$
\square \varphi+m^{2} \varphi=0,
$$


where $\square$ is the d'Alembert operator in $d+1$ dimensions: $\square:=$ $\partial^{2} / \partial t^{2}-\sum_{i=1}^{d}\left(\partial^{2} / \partial x_{i}^{2}\right)$. Considering the momenta $\pi:=\partial \varphi / \partial t$, one can rewrite (14) as a first-order system:

$$
\begin{aligned}
& \frac{\partial \varphi}{\partial t}=\pi, \\
& \frac{\partial \pi}{\partial t}=-\left(m^{2}-\Delta\right) \varphi,
\end{aligned}
$$

where $\Delta:=\sum_{i=1}^{d}\left(\partial^{2} / \partial x_{i}^{2}\right)$. The evolution equations (15) can be written in Hamiltonian form:

$$
\begin{aligned}
& \frac{\partial \varphi}{\partial t}=\{\varphi, H\}, \\
& \frac{\partial \pi}{\partial t}=\{\pi, H\},
\end{aligned}
$$

with Hamiltonian function given by

$$
H=\frac{1}{2} \int d^{d} x\left(\pi^{2}+\varphi\left(m^{2}-\Delta\right) \varphi\right)
$$

and Poisson bracket defined in the usual way, involving functional derivatives.

The operator $m^{2}-\Delta$ plays an important role in what follows. The following proposition collects some properties of this operator $[2,6,13]$.

Proposition 7. (i) The differential operator $m^{2}-\Delta$ is a continuous linear operator in the real Schwartz space $\mathcal{S}\left(\mathbb{R}^{d}\right)$, with respect to the nuclear topology. The same is true for the inverse operator $\left(m^{2}-\Delta\right)^{-1}$. When considered as an operator on the complex Schwartz space $\mathcal{S}^{\mathbb{C}}\left(\mathbb{R}^{d}\right), m^{2}-\Delta$ defines a selfadjoint positive operator on $L^{2}\left(\mathbb{R}^{d}\right)$. The operators $\left(m^{2}-\Delta\right)^{l / 2^{j}}$, $l, j \in \mathbb{N}$, are densely defined in $L^{2}\left(\mathbb{R}^{d}\right)$ and enjoy the same above-mentioned properties.

(ii) The inverse operator $\left(m^{2}-\Delta\right)^{-1}$ is bounded, self-adjoint, and positive on $L^{2}\left(\mathbb{R}^{d}\right)$. The operators $\left(m^{2}-\Delta\right)^{-l / 2^{j}}, l, j \in \mathbb{N}$, are well defined and enjoy the same properties.

(iii) The operators $\left(m^{2}-\Delta\right)^{ \pm l / 2^{j}}, l, j \in \mathbb{N}$, are strictly positive on $L^{2}\left(\mathbb{R}^{d}\right)$; that is, $\left\langle\psi,\left(m^{2}-\Delta\right)^{ \pm l / 2^{j}} \psi\right\rangle=0$ for $\psi$ in the domain implies $\psi=0$.

The evolution equations (15) are easily solved in an appropriate Hilbert space. We follow here [1, 2]. Formally, the fundamental solution of (15) is given by the kernel of the evolution operator

$$
\Theta_{m}(t)=\exp \left(\left(\begin{array}{cc}
0 & 1 \\
-\left(m^{2}-\Delta\right) & 0
\end{array}\right) t\right)
$$

that one obtains by exponentiation of the operator $\left(\begin{array}{cc}0 & 1 \\ -\left(m^{2}-\Delta\right) & 0\end{array}\right)$ in system (15). The rigorous treatment of this result, however, requires an appropriate Hilbert structure which we now describe. Let $\widetilde{\mathscr{H}}_{m}^{ \pm}$be the Hilbert spaces obtained by completion of $\delta^{\mathbb{C}}\left(\mathbb{R}^{d}\right)$ with respect to the inner products. Consider

$$
(f, g) \longmapsto\left\langle f,\left(m^{2}-\Delta\right)^{ \pm 1 / 2} g\right\rangle .
$$

The operator

$$
i\left(\begin{array}{cc}
0 & 1 \\
-\left(m^{2}-\Delta\right) & 0
\end{array}\right)
$$

is densely defined in $\widetilde{\mathscr{H}}_{m}^{+} \oplus \widetilde{\mathscr{H}}_{m}^{-}$and is self-adjoint [2]. Then, (18) gives us a well defined unitary representation of $\mathbb{R}$ on $\widetilde{\mathscr{H}}_{m}^{+} \oplus \widetilde{\mathscr{H}}_{m}^{-}$, which is strongly continuous. It can be shown [2] that the operators $\Theta_{m}(t)$ (18) preserve the real subspaces of $\widetilde{\mathscr{H}}_{m}^{+} \oplus \widetilde{\mathscr{H}}_{m}^{-}$. Thus, the operators $\Theta_{m}(t)$ are orthogonal on the real Hilbert space $\mathscr{H}_{m}^{+} \oplus \mathscr{H}_{m}^{-}$, where $\mathscr{H}_{m}^{ \pm}$is the completion of the real Schwartz space $\mathcal{S}\left(\mathbb{R}^{d}\right)$ with respect to (19). The dynamics of the free field of mass $m$ is therefore well defined on the space $\mathscr{H}_{m}^{+} \oplus \mathscr{H}_{m}^{-}$, which can then be taken as the appropriate classical phase space for this system. The symplectic form in this linear space is given by

$$
\Omega\left(\left(\varphi_{1}, \pi_{1}\right),\left(\varphi_{2}, \pi_{2}\right)\right)=\left\langle\varphi_{1}, \pi_{2}\right\rangle-\left\langle\varphi_{2}, \pi_{1}\right\rangle,
$$

where the map

$$
\mathscr{H}_{m}^{+} \oplus \mathscr{H}_{m}^{-} \ni(\varphi, \pi) \longmapsto\langle\varphi, \pi\rangle=\int_{\mathbb{R}^{d}} \varphi \pi d^{d} x
$$

is well defined and continuous.

Let $\langle\langle,\rangle\rangle_{-m}$ be $\mathscr{H}_{m}^{+} \oplus \mathscr{H}_{m}^{-}$inner product. The form $\Omega(21)$ can be written as

$$
\begin{gathered}
\Omega\left(\left(\varphi_{1}, \pi_{1}\right),\left(\varphi_{2}, \pi_{2}\right)\right)=\left\langle\left\langle\left(\varphi_{1}, \pi_{1}\right),\right.\right. \\
\left(\begin{array}{cc}
\left(m^{2}-\Delta\right)^{-1 / 2} & 0 \\
0 & \left(m^{2}-\Delta\right)^{-1 / 2}
\end{array}\right) \\
\left.\left.\cdot\left(\begin{array}{cc}
0 & 1 \\
-\left(m^{2}-\Delta\right) & 0
\end{array}\right)\left(\varphi_{2}, \pi_{2}\right)\right\rangle\right\rangle_{-m} .
\end{gathered}
$$

It is then clear that the group $\Theta_{m}(t)$ preserves the symplectic form given that $\Theta_{m}(t)$ is orthogonal in $\mathscr{H}_{m}^{+} \oplus \mathscr{H}_{m}^{-}, \forall t$, and commutes with

$$
\left(\begin{array}{cc}
\left(m^{2}-\Delta\right)^{-1 / 2} & 0 \\
0 & \left(m^{2}-\Delta\right)^{-1 / 2}
\end{array}\right)
$$

and with

$$
\left(\begin{array}{cc}
0 & 1 \\
-\left(m^{2}-\Delta\right) & 0
\end{array}\right) .
$$

Let us consider the evolution of the kinematical observables $F_{c,(f, g)}$ :

$$
F_{c,(f, g)}(\varphi, \pi):=c+\langle f, \varphi\rangle+\langle g, \pi\rangle,
$$


where $c \in \mathbb{R}$ and $f, g \in \mathcal{S}\left(\mathbb{R}^{d}\right)$. For each $t \in \mathbb{R}$, let $F_{c,(f, g)}^{t}$ be the pull-back of $F_{c,(f, g)}$ :

$$
F_{c,(f, g)}^{t}(\varphi, \pi):=F_{c,(f, g)}\left(\Theta_{m}(t)(\varphi, \pi)\right) .
$$

It is clear that $F_{c,(f, g)}^{t}=F_{c, S_{m}(t)(f, g)}, \forall t \in \mathbb{R}$, where

$$
S_{m}(t):=\exp \left(\left(\begin{array}{cc}
0 & -\left(m^{2}-\Delta\right) \\
1 & 0
\end{array}\right) t\right)
$$

is an orthogonal operator on the real Hilbert space $\mathscr{H}_{m}^{-} \oplus \mathscr{H}_{m}^{+}$. The linear space $\mathbb{R} \oplus\left(\mathscr{H}_{m}^{-} \oplus \mathscr{H}_{m}^{+}\right)$of test functions is a Lie algebra under the Lie bracket

$$
\begin{aligned}
& {\left[(c,(f, g)),\left(c^{\prime},\left(f^{\prime}, g^{\prime}\right)\right)\right]} \\
& \quad=\left(\left\langle f, g^{\prime}\right\rangle-\left\langle g, f^{\prime}\right\rangle,(0,0)\right) .
\end{aligned}
$$

This algebra is obviously isomorphic to the algebra of kinematical functions (26) under the Poisson bracket. The above result shows that the dynamics is implemented on the kinematical algebra as a group of linear automorphisms. The group $S_{m}(t)(28)$ is an orthogonal representation of $\mathbb{R}$ on $\mathscr{H}_{m}^{-} \oplus \mathscr{H}_{m}^{+}$.

\section{Unitary Implementation of Linear Symplectomorphisms}

In this section we discuss the question of unitary implementation of linear canonical transformations in the context of Gaussian representations of the Weyl relations for the real scalar field. We follow [1], although considering real Hilbert spaces instead of complex ones, in the description of the classical phase space and kinematical algebra.

Let $A$ be a linear transformation on $\mathcal{S}\left(\mathbb{R}^{d}\right) \oplus \mathcal{S}\left(\mathbb{R}^{d}\right)$, continuous in the nuclear topology and with continuous inverse. Like in finite dimensions, we will say that $A$ is symplectic if $A$ preserves the symplectic matrix $\mathbb{V}=\left(\begin{array}{cc}0 & 1 \\ -1 & 0\end{array}\right)$ in $\delta\left(\mathbb{R}^{d}\right) \oplus$ $\mathcal{S}\left(\mathbb{R}^{d}\right)$. We just saw in the last section that the classical evolution of the free field of mass $m$ is determined by a oneparameter group of linear symplectic transformations $S_{m}(t)$ (28). To quantize the system, one needs a representation of the Weyl relations allowing the unitary implementation of this group. In more precise terms, one looks for a representation $\left(\mathscr{H}_{m}, \mathscr{W}_{m}\right)$ of the Weyl relations by Weyl operators $\mathscr{W}_{m}(f, g)$, $f, g \in \delta\left(\mathbb{R}^{d}\right)$, on a (complex) Hilbert space $\mathscr{H}_{m}$ such that there exists a group of unitary transformations $T_{m}(t)$ : $\mathscr{H}_{m} \rightarrow \mathscr{H}_{m}, t \in \mathbb{R}$, satisfying

$$
T_{m}(t) \mathscr{W}_{m}(f, g) T_{m}(t)^{-1}=\mathscr{W}_{m}\left(S_{m}(t)(f, g)\right) .
$$

For the free field, (30) is satisfied by a Gaussian representation. In fact, the (equivalence class of the) representation $\left(\mathscr{H}_{m}, \mathscr{W}_{m}\right)$ is uniquely determined by the dynamics, that is, by (30) and by the natural conditions of positivity of the quantum Hamiltonian and unicity of the vacuum. This result is based on the theorem below [1]. Before presenting the theorem, let us illustrate the nontriviality of the quantization process for symplectomorphisms (in general for observables outside the kinematical algebra).

Let $(\mathscr{H}, \mathscr{W})$ be a continuous and irreducible representation of the Weyl relations and $A$ a linear symplectomorphism. We can define a new continuous and irreducible representation $\left(\mathscr{H}, \mathscr{W}_{A}\right)$ on the same Hilbertspace $\mathscr{H}$ by

$$
\mathscr{W}_{A}(f, g):=\mathscr{W}(A(f, g)) .
$$

In finite dimensions the Stone-von Neumann theorem shows that $\mathscr{W}$ and $\mathscr{W}_{A}$ are unitarily equivalent and therefore guarantees the existence of a unitary operator $U(A)$ corresponding to the quantization of $A$. In infinite dimensions, however, the representations $\mathscr{W}$ and $\mathscr{W}_{A}$ are not necessarily equivalent and therefore the quantization of a given canonical transformation $A$ does not necessarily exist for an arbitrary representation $\mathscr{W}$. Gaussian representations give us good examples of this fact, as we now show. Let $A_{\sigma}$ be the symplectic transformation given by

$$
(f, g) \longmapsto\left(\sigma^{1 / 2} f, \sigma^{-1 / 2} g\right), \quad \sigma \in \mathbb{R}^{+}, \sigma \neq 1 .
$$

For convenience of notation, let us denote by $\mathscr{W}^{\mathrm{C}}$ the Gaussian representation on the Hilbert space $L^{2}\left(\mathcal{S}^{\prime}\left(\mathbb{R}^{d}\right), \mu\right)$, defined by the Gaussian measure $\mu$ of covariance $C$ : $\mathcal{S}\left(\mathbb{R}^{d}\right) \rightarrow \mathcal{S}\left(\mathbb{R}^{d}\right)$. Let us consider the new representation

$$
\mathscr{W}_{A_{\sigma}}^{C}(f, g):=\mathscr{W}^{C}\left(\sigma^{1 / 2} f, \sigma^{-1 / 2} g\right) .
$$

For every $f, g \in \mathcal{S}\left(\mathbb{R}^{d}\right)$ one has

$$
\left\langle 1, \mathscr{W}_{A_{\sigma}}^{C}(f, g) 1\right\rangle=\left\langle 1, \mathscr{W}^{\sigma C}(f, g) 1\right\rangle
$$

and therefore the two representations $\mathscr{W}_{A_{\sigma}}^{C}$ and $\mathscr{W}^{\sigma C}$ are unitarily equivalent (see Section 2.2). On the other hand, the representations $\mathscr{W}^{C}$ and $\mathscr{W}^{\sigma C}$ are not equivalent given that the corresponding measures are not mutually absolutely continuous. So, one may conclude that $A_{\sigma}$ does not admit a quantization compatible with any Gaussian representation of the Weyl relations.

We now define the group of linear symplectomorphisms that does admit a natural quantization for a given Gaussian representation. Let then $C$ be a covariance operator on $\mathcal{S}\left(\mathbb{R}^{d}\right)$ and consider the Gaussian representation $\left(L^{2}\left(\mathcal{S}^{\prime}\left(\mathbb{R}^{d}\right), \mu\right), \mathscr{W}\right)$ of the Weyl relations, defined by $C$, where $\mu$ is the Gaussian measure of covariance $C$. Note that the representation $\mathscr{W}(f, g)$ can be continuously extended to all $(f, g) \in \mathscr{H}_{C} \oplus$ $\mathscr{H}_{\mathrm{C}^{-1}}$, where $\mathscr{H}_{\mathrm{C}} \oplus \mathscr{H}_{\mathrm{C}^{-1}}$ is the real Hilbert space one obtains by completion of $\delta\left(\mathbb{R}^{d}\right) \oplus \mathcal{S}\left(\mathbb{R}^{d}\right)$ with respect to the inner product,

$$
\begin{aligned}
& \left\langle\left\langle(f, g),\left(f^{\prime}, g^{\prime}\right)\right\rangle\right\rangle_{C} \\
& \quad:=\langle f,(2 C) f\rangle+\left\langle g,(2 C)^{-1} g\right\rangle,
\end{aligned}
$$

as follows from considerations in Section 2.2. 
Consider the group of (linear) orthogonal symplectomorphisms, that is, the group $G_{C}$ of linear operators $A$ on $\mathscr{H}_{C} \oplus$ $\mathscr{H}_{C^{-1}}$, such that

$$
\begin{gathered}
\left\langle\left\langle A(f, g), A\left(f^{\prime}, g^{\prime}\right)\right\rangle\right\rangle_{C}=\left\langle\left\langle(f, g),\left(f^{\prime}, g^{\prime}\right)\right\rangle\right\rangle_{C}, \\
\left\langle A(f, g), \mathbb{J}\left(f^{\prime}, g^{\prime}\right)\right\rangle=\left\langle(f, g), \mathbb{J}\left(f^{\prime}, g^{\prime}\right)\right\rangle,
\end{gathered}
$$

where

$$
\left\langle(f, g), \mathbb{J}\left(f^{\prime}, g^{\prime}\right)\right\rangle:=\left\langle f, g^{\prime}\right\rangle-\left\langle f^{\prime}, g\right\rangle .
$$

In what follows we will consider on $G_{C}$ the topology induced from the strong topology associated with $\langle\langle,\rangle\rangle_{C}$ norm.

Theorem 8. Let $C$ be a covariance operator on $\mathcal{S}\left(\mathbb{R}^{d}\right), \mu$ the corresponding Gaussian measure on $\mathcal{S}^{\prime}\left(\mathbb{R}^{d}\right)$, and $\mathscr{W}$ the associated Gaussian representation of the Weyl relations. On $L^{2}\left(\mathcal{S}^{\prime}\left(\mathbb{R}^{d}\right), \mu\right)$ there is a unique strongly continuous unitary representation $U$ of the group $G_{C}$ such that

$$
\begin{aligned}
U(A) \mathscr{W}(f, g) U(A)^{-1}= & \mathscr{W}(A(f, g)), \\
& \forall A \in G_{C}, \forall f, g \in \mathcal{S}\left(\mathbb{R}^{d}\right), \\
U(A) 1= & 1, \quad \forall A \in G_{C} .
\end{aligned}
$$

To prove the theorem, let us start by showing uniqueness. Suppose that $U$ and $\widetilde{U}$ are two such representations of $G_{C}$. Then, for all $A \in G_{C}, \widetilde{U}(A)^{-1} U(A)$ commutes with $\mathscr{W}(f, g)$, $\forall f, g$, which implies that $\widetilde{U}(A)^{-1} U(A)$ is proportional to the identity, given the irreducibility of $\mathscr{W}$. This still does not prove that $\widetilde{U}$ coincide exactly with $U$, but since by (39) $\widetilde{U}(A)^{-1} U(A) 1=1$, we conclude that $\widetilde{U}(A)=U(A), \forall A$. Let us show that a representation exists. For every $A \in G_{C}, \mathscr{W}_{A}$ defined by (31) is irreducible and continuous with respect to $\langle\langle,\rangle\rangle_{C}$, since $A$ is orthogonal. The crucial fact is that since the expectation values $\langle 1, \mathscr{W}(f, g) 1\rangle$ depend only on the inner product $\langle\langle,\rangle\rangle_{C}$ (see Section 2.2 ), one gets that $\left\langle 1, \mathscr{W}_{A} 1\right\rangle=$ $\langle 1, \mathscr{W} 1\rangle, \forall A \in G_{C}$. This in turn implies that $\mathscr{W}$ and $\mathscr{W}_{A}$ are equivalent representations, $\forall A \in G_{C}$. One can show that the unitary operator $U(A)$ on $L^{2}\left(\mathcal{S}^{\prime}\left(\mathbb{R}^{d}\right), \mu\right)$ defined by

$$
\sum_{k} \lambda_{k} \mathscr{W}(f, 0) 1 \stackrel{U(A)}{\longmapsto} \sum_{k} \lambda_{k} \mathscr{W}\left(A\left(f_{k}, 0\right)\right) 1, \quad \lambda_{k} \in \mathbb{C}
$$

satisfies both (38) and (39). From (40) it follows immediately that the operators $U(A)$ are a representation of $G_{C}$. The proof of the continuity of this representation also presents no significative difficulty (see, e.g., [1]).

\section{Quantization of Dynamics: Free Field Measure}

In this section we present the quantization of the free real scalar field of mass $m$ in $d+1$ dimensions, following $[1,2,6]$. Recall that the phase space $\mathscr{H}_{m}^{+} \oplus \mathscr{H}_{m}^{-}$and the space of test functions $\mathscr{H}_{m}^{-} \oplus \mathscr{H}_{m}^{+}$for the classical field of mass $m$ are naturally equipped with a real Hilbert space structure and that the classical evolution acts by orthogonal transformations. Using the inner product on $\mathscr{H}_{m}^{-}$one can define a Gaussian representation of the Weyl relations for which the quantization of the dynamics is guaranteed by Theorem 8.

As we saw in Section 3, Proposition 7, the operator

$$
C_{m}:=\frac{1}{2}\left(m^{2}-\Delta\right)^{-1 / 2}
$$

associated with the classical Hamiltonian possesses all the properties of a covariance operator on $\delta\left(\mathbb{R}^{d}\right)$. Let $\mu^{m}$ be the Gaussian measure on $\mathcal{S}^{\prime}\left(\mathbb{R}^{d}\right)$ of covariance $C_{m}$. The measure $\mu^{m}$, or equivalently the covariance $C_{m}$, defines a (cyclic) Gaussian representation $\left(L^{2}\left(\mathcal{S}^{\prime}\left(\mathbb{R}^{d}\right), \mu^{m}\right), \mathscr{W}_{m}\right)$ of the Weyl relations such that

$$
\begin{aligned}
& \left\langle 1, \mathscr{W}_{m}(f, g) 1\right\rangle=\exp \left(-\frac{1}{4}\left(\left\langle f,\left(m^{2}-\Delta\right)^{-1 / 2} f\right\rangle\right.\right. \\
& \left.\left.+\left\langle g,\left(m^{2}-\Delta\right)^{1 / 2} g\right\rangle\right)\right) .
\end{aligned}
$$

Explicitly, the representation is defined by the operators $\mathcal{U}_{m}(f):=\mathscr{W}_{m}(f, 0), \mathscr{V}_{m}(g):=\mathscr{W}_{m}(0, g)$ :

$$
\begin{aligned}
& \left(\mathscr{U}_{m}(f) \psi\right)(\phi)=e^{-i \phi(f)} \psi(\phi), \\
& \left(\mathscr{V}_{m}(g) \psi\right)(\phi)=e^{-\left\langle g, C_{m}^{-1} g\right\rangle / 4} e^{\phi\left(C_{m}^{-1} g\right) / 2} \psi(\phi-g),
\end{aligned}
$$

where $\psi \in L^{2}\left(\mathcal{S}^{\prime}\left(\mathbb{R}^{d}\right), \mu^{m}\right)$.

The quantization of the coordinate functions

$$
\begin{array}{ll}
\boldsymbol{\varphi}_{f}(\varphi, \pi):=\langle f, \varphi\rangle, & f \in \mathcal{S}\left(\mathbb{R}^{d}\right), \\
\boldsymbol{\pi}_{g}(\varphi, \pi):=\langle g, \pi\rangle, & g \in \mathcal{S}\left(\mathbb{R}^{d}\right)
\end{array}
$$

that generate the kinematical algebra is given by the generators of $\mathscr{U}_{m}$ and $\mathscr{V}_{m}$ as follows. There is a dense subspace $\mathscr{D} \subset L^{2}\left(\mathcal{S}^{\prime}\left(\mathbb{R}^{d}\right), \mu^{m}\right)$ and operators $Q_{m}\left(\varphi_{f}\right)$ and $\mathcal{Q}_{m}\left(\pi_{g}\right)$ (essentially) are self-adjoint on $\mathscr{D}$ such that [2]

$$
\begin{aligned}
& \mathscr{U}_{m}(s f)=\exp \left(-i s Q_{m}\left(\boldsymbol{\varphi}_{f}\right)\right), \\
& \mathscr{V}_{m}(s g)=\exp \left(-i s Q_{m}\left(\boldsymbol{\pi}_{g}\right)\right), \quad \forall s \in \mathbb{R} .
\end{aligned}
$$

On $\mathscr{D}$, the canonical commutation relations

$$
\left[Q_{m}\left(\boldsymbol{\varphi}_{f}\right), Q_{m}\left(\boldsymbol{\pi}_{g}\right)\right]=i\langle f, g\rangle \mathbf{1}
$$

are satisfied.

As we saw, the representation can be continuously extended to all $(f, g) \in \mathscr{H}_{C_{m}} \oplus \mathscr{H}_{C_{m}^{-1}}$. With $C_{m}$ given by (41), this Hilbert space is precisely the test function space $\mathscr{H}_{m}^{-} \oplus \mathscr{H}_{m}^{+}$. We will stick to this latter notation.

The fundamental aspect of the representation $\mathscr{W}_{m}$ is that of allowing a quantization of the dynamics for the field of mass $m$. As we saw in Section 3, the kinematical observables $F_{c,(f, q)}$, or equivalently the test functions $(f, g)$, evolve under the action of a group $S_{m}(t)$ (28) of orthogonal linear symplectomorphisms on $\mathscr{H}_{m}^{-} \oplus \mathscr{H}_{m}^{+}$. We thus have the following fundamental result as a corollary of Theorem 8 . 
Corollary 9. On $L^{2}\left(\mathcal{S}^{\prime}\left(\mathbb{R}^{d}\right), \mu^{m}\right)$ is defined a continuous unitary representation $T_{m}(t)$ of $\mathbb{R}$ such that

$$
\begin{aligned}
T_{m}(t) \mathscr{W}_{m}(f, g) T_{m}(t)^{-1} & =\mathscr{W}_{m}\left(S_{m}(t)(f, g)\right), \\
\forall t \in \mathbb{R}, \forall(f, g), & \\
T_{m}(t) 1 & =1, \quad \forall t .
\end{aligned}
$$

This result gives a quantization of the dynamics or a quantization $\mathbb{Q}_{m}(H)$ of the Hamiltonian defined by

$$
T_{m}(t)=: \exp \left(i t \mathbb{Q}_{m}(H)\right) .
$$

It can be shown $[1,2,6]$ that the quantum Hamiltonian has nonnegative spectrum and that the zero eigenvalue is nondegenerate, establishing the interpretation of the cyclic vector 1 as the vacuum. It can also be shown that the quantization of the free field of mass $m$ above is unique [1], that is, given a cyclic representation $\mathscr{W}$ of the Weyl relations such that there exists a unitary one-parameter group $T(t)=$ : $\exp (i t \mathbb{Q}(H))$ with nonnegative generator $\mathbb{Q}(H)$ and such that (47) are satisfied; one can find a unitary operator $U$ relating both $\mathscr{W}$ to $\mathscr{W}_{m}$ and $T$ to $T_{m}$ :

$$
\begin{aligned}
U \mathscr{W}(f, g) U^{-1} & =\mathscr{W}_{m}(f, g), \quad \forall f, g, \\
U T(t) U^{-1} & =T_{m}(t), \quad \forall t .
\end{aligned}
$$

\section{Invariance and Ergodicity of the Action of the Euclidean Group}

In this section we show that the measure $\mu^{m}$ for the free field is invariant and ergodic with respect to the natural action of the Euclidean group on $\mathbb{R}^{d}$. The invariance gives us a unitary representation of this symmetry group on the quantum Hilbert space, and ergodicity implies that the vacuum is the only invariant state (see, e.g., [14]). The invariance and ergodicity of the measure $\mu^{m}, \forall m$, also imply that two measures $\mu^{m}$ and $\mu^{m^{\prime}}, m \neq m^{\prime}$, are supported on disjoint sets (see, e.g., [14]), which in turn leads immediately to the nonequivalence of the corresponding representations of the Weyl relations.

The Euclidean group $\mathscr{E}$ on $\mathbb{R}^{d}$ acts on $\delta\left(\mathbb{R}^{d}\right)$ by

$$
f \longmapsto f_{\gamma}: f_{\gamma}(x)=f\left(\gamma^{-1} x\right),
$$

where $\gamma \in \mathscr{E}$ and $\gamma x$ denotes the natural action of $\mathscr{E}$ on $\mathbb{R}^{d}$. The action on $\delta\left(\mathbb{R}^{d}\right)$ induces an action $\varphi$ on $\mathcal{S}^{\prime}\left(\mathbb{R}^{d}\right)$ :

$$
\left(\varphi_{\gamma} \phi\right)(f)=\phi\left(f_{\gamma}\right) .
$$

The invariance of the measure $\mu^{m}$ follows immediately from the invariance of the covariance $C_{m}=(1 / 2)\left(m^{2}-\Delta\right)^{-1 / 2}$.

Proposition 10. The measure $\mu^{m}$ is $\mathscr{E}$-invariant, for any $m$.

One thus has a unitary action $U$ of $\mathscr{E}$ on $L^{2}\left(\mathcal{S}^{\prime}\left(\mathbb{R}^{d}\right), \mu^{m}\right)$ :

$$
(U(\gamma) \psi)(\phi)=\psi\left(\varphi_{\gamma}^{-1} \phi\right), \quad \psi \in L^{2}\left(\mathcal{S}^{\prime}\left(\mathbb{R}^{d}\right), \mu^{m}\right) .
$$

Let us consider the subgroup (isomorphic to $\mathbb{R}$ ) of $\mathscr{E}$ of all translations in a fixed direction (for instance, parallel to $x_{1}$ axis):

$$
\left(x_{1}, x_{2}, \ldots, x_{d}\right) \longmapsto\left(x_{1}+y, x_{2}, \ldots, x_{d}\right), \quad y \in \mathbb{R} .
$$

Definition 11. Given a probability space $M$ with measure $\mu$, a measure preserving action of $\mathbb{R}$ is said to be mixing if the corresponding unitary action $U$ on $L^{2}(M, \mu)$ satisfies

$$
\begin{aligned}
& \lim _{y \rightarrow \infty}\left\langle\psi^{\prime}, U(y) \psi\right\rangle=\left\langle\psi^{\prime}, 1\right\rangle\langle 1, \psi\rangle, \\
& \forall \psi^{\prime}, \psi \in L^{2}(M, \mu) .
\end{aligned}
$$

It follows from (54) that any invariant element of $L^{2}(M, \mu)$ is constant a.e., and therefore mixing implies ergodicity $[15$, 16].

Proposition 12. The action (52) of the subgroup (53) is mixing.

In fact, by linearity and continuity, it is sufficient to verify (54) for the functions of the form $e^{-i \phi(f)}$ whose linear span is dense. For those functions

$$
\begin{aligned}
& \left\langle e^{-i \phi\left(f^{\prime}\right)}, U(y) e^{-i \phi(f)}\right\rangle=\int e^{-i \phi\left(f_{-y}-f^{\prime}\right)} d \mu^{m} \\
& =\exp \left(-\frac{1}{2}\left\langle\left(f_{-y}-f^{\prime}\right), C_{m}\left(f_{-y}-f^{\prime}\right)\right\rangle\right) \\
& =\left\langle e^{-i \phi\left(f^{\prime}\right)}, 1\right\rangle\left\langle 1, e^{-i \phi(f)}\right\rangle \cdot \exp \left(\frac{1}{2}\right. \\
& \cdot \int f^{\prime}\left(x_{1}, \ldots, x_{d}\right)\left(m^{2}-\Delta\right)^{-1 / 2} \\
& \left.\cdot f\left(x_{1}+y, \ldots, x_{d}\right) d^{d} x\right) .
\end{aligned}
$$

By Fourier transform, the integral in exponent on (55) can be written as

$$
\int e^{-i k_{1} y}\left(\int \frac{\widetilde{f^{\prime}}(k) \tilde{f}(k)}{\left(m^{2}+k^{2}\right)^{1 / 2}} d k_{2} \cdots d k_{d}\right) d k_{1} .
$$

The integral (56), seen as a function of $y$, is the Fourier transform of a function in $\mathcal{S}(\mathbb{R})$. By the Riemann-Lebesgue lemma, (56) goes to zero in the limit $y \rightarrow \infty$. Going back to (55), we conclude that the action is mixing.

The subgroup (53), and consequently any subgroup that contains it, acts therefore ergodically. Note also that $\mathscr{E}$ invariance of the measure implies that the subgroup of translations in any fixed direction acts ergodically. We conclude that (i) the vacuum is the only state invariant under the action of translations of the type (53) and (ii) two measures $\mu^{m}$ and $\mu^{m^{\prime}}, m \neq m^{\prime}$, are supported on mutually disjoint sets, implying the nonunitary equivalence of the corresponding free field representations.

\section{Covariant Formulation}

We will now consider the relativistic invariance properties of the free field quantization, showing explicitly how a covariant 
formulation can be obtained from the above canonical quantization. Although presented here in heuristic form, one can give a precise meaning to the results in this section (see [1] for a rigorous approach). We start by considering the evolution of the quantum operators $Q_{m}\left(\varphi_{f}\right)$ and $Q_{m}\left(\pi_{g}\right)$ (see (45)), which is simply dictated by the classical evolution of test functions. One can then fully reconstruct a relativistic quantum field obeying an appropriate quantum version of the classical Klein-Gordon equation.

Let us consider the time-dependent Weyl operators:

$$
\begin{aligned}
\mathscr{W}_{m}^{t} & :=T_{m}(t) \mathscr{W}_{m}(f, g) T_{m}(t)^{-1} \\
& =\mathscr{W}_{m}\left(S_{m}(t)(f, g)\right)
\end{aligned}
$$

To simplify the notation we introduce time-dependent field operators $\widehat{\varphi}_{t}(f)$ and $\widehat{\pi}_{t}(g)$ such that $\widehat{\varphi}_{0}(f)=\widehat{Q}_{m}\left(\varphi_{f}\right)$ and $\hat{\pi}_{0}(g)=Q_{m}\left(\pi_{g}\right)$. These operators describe the evolution of the corresponding time-zero operators and are defined by the following condition:

$$
\begin{aligned}
\mathscr{W}_{m}^{t}(s f, r g)=\exp \left[-i s \widehat{\varphi}_{t}(f)-i \widehat{\pi}_{t}(g)\right], & \\
& \forall s, r \in \mathbb{R}, \forall f, g \in \mathcal{S}\left(\mathbb{R}^{d}\right) .
\end{aligned}
$$

We can rewrite this expression in the more convenient form

$$
\mathscr{W}_{m}^{t}(s f, r g)=\exp \left[-i\left(\begin{array}{ll}
\widehat{\varphi}_{t} & \hat{\pi}_{t}
\end{array}\right)\left(\begin{array}{c}
s f \\
r g
\end{array}\right)\right],
$$

where $\left(\widehat{\varphi}_{t} \widehat{\pi}_{t}\right)\left(\begin{array}{l}f \\ g\end{array}\right)$ stands for $\widehat{\varphi}_{t}(f)+\widehat{\pi}_{t}(g)$. Equation (57) then translates to

$$
\mathscr{W}_{m}^{t}(s f, r g)=\exp \left[-i\left(\begin{array}{ll}
\widehat{\varphi}_{0} & \widehat{\pi}_{0}
\end{array}\right) S_{m}(t)\left(\begin{array}{c}
s f \\
r g
\end{array}\right)\right] .
$$

From (59) and (60) it follows that

$$
\begin{aligned}
& \widehat{\varphi}_{t}(f)=\left(\begin{array}{ll}
\widehat{\varphi}_{0} & \widehat{\pi}_{0}
\end{array}\right) S_{m}(t)\left(\begin{array}{l}
f \\
0
\end{array}\right), \\
& \widehat{\pi}_{t}(g)=\left(\begin{array}{ll}
\widehat{\varphi}_{0} & \widehat{\pi}_{0}
\end{array}\right) S_{m}(t)\left(\begin{array}{l}
0 \\
g
\end{array}\right) .
\end{aligned}
$$

To arrive at the relativistic formulation we will obtain first the second-order differential equation for $\widehat{\varphi}_{t}(f)$. Taking into account that (see Section 3)

$$
\frac{d S_{m}(t)}{d t}=S_{m}(t)\left(\begin{array}{cc}
0 & -\left(m^{2}-\Delta\right) \\
1 & 0
\end{array}\right),
$$

one gets, after using again (61),

$$
\begin{aligned}
\frac{d}{d t} \widehat{\varphi}_{t}(f) & =\widehat{\pi}_{t}(f), \\
\frac{d}{d t} \widehat{\pi}_{t}(g) & =-\widehat{\varphi}_{t}\left(\left(m^{2}-\Delta\right) g\right) .
\end{aligned}
$$

The second-order equation now follows:

$$
\frac{d^{2}}{d t^{2}} \widehat{\varphi}_{t}(f)+\widehat{\varphi}_{t}\left(\left(m^{2}-\Delta\right) f\right)=0 .
$$

We now introduce space-time averages. For any $F(t, x) \in$ $\mathcal{S}\left(\mathbb{R}^{d+1}\right)$, the integral

$$
\int \widehat{\varphi}_{t}(F(t, \cdot)) d t
$$

defines a self-adjoint operator [1], which we will denote by $\Phi(F)$. The map $\Phi$ from $\mathcal{S}\left(\mathbb{R}^{d+1}\right)$ to self-adjoint operators on $L^{2}\left(\mathcal{S}^{\prime}\left(\mathbb{R}^{d}\right), \mu^{m}\right)$ is interpreted as the relativistic quantum field and satisfies an equation analogous to the classical KleinGordon equation:

$$
\Phi\left(\left(\square+m^{2}\right) F\right)=0 .
$$

One can show that there exists a unitary representation $\Gamma$ of the Poincaré group such that for any Poincaré transformation $\Lambda$ one has

$$
\begin{gathered}
\Gamma(\Lambda) 1=1, \\
\Gamma(\Lambda) \Phi(F) \Gamma(\Lambda)^{-1}=\Phi\left(F_{\Lambda}\right),
\end{gathered}
$$

where $F_{\Lambda}(t, x)=F\left(\Lambda^{-1}(t, x)\right)$.

\section{Local Properties of the Support of the Free Field Measure}

We will now present a characterization of the support of the free field measure $\mu^{m}$ defined by the covariance (41), following [8-11]. The result in question is a consequence of the so-called Minlos' theorem, which for the case of Gaussian measures in $\mathcal{S}^{\prime}\left(\mathbb{R}^{d}\right)$ can be stated as follows (see $\left.[9,11,14]\right)$.

Theorem 13 (Minlos). Let (, ) be a continuous inner product on $\mathcal{S}\left(\mathbb{R}^{d}\right)$ and $\mathscr{H}$ the corresponding completion of $\mathcal{S}\left(\mathbb{R}^{d}\right)$. Let $H$ be an injective Hilbert-Schmidt operator on the Hilbert space $\mathscr{H}$ such that $\mathcal{S}\left(\mathbb{R}^{d}\right) \subset H \mathscr{H}$ and $H^{-1}: \mathcal{S}\left(\mathbb{R}^{d}\right) \rightarrow \mathscr{H}$ is a continuous map. Let $(,)_{1}$ be the inner product on $\mathcal{S}\left(\mathbb{R}^{d}\right)$ defined by $(f, g)_{1}=\left(H^{-1} f, H^{-1} g\right)$. Then, the Gaussian measure on the dual space $\mathcal{S}^{\prime}\left(\mathbb{R}^{d}\right)$ with covariance $($, ) is supported on the subspace of $\mathcal{S}^{\prime}\left(\mathbb{R}^{d}\right)$ of those functionals which are continuous with respect to the topology defined by $(,)_{1}$.

Let us then apply Theorem 13 to the Gaussian measure defined by the covariance operator (41), so that we can obtain sets of measure one. We will show that the set of distributions supporting the measure are such that the action of the operator $\left(1+x^{2}\right)^{-\alpha}\left(m^{2}-\Delta\right)^{-\beta}$ produces $L^{2}\left(\mathbb{R}^{d}\right)$ elements, for $\alpha>d / 4$ and $\beta>(d-1) / 4$. To prove this, let us consider the operators

$$
\widetilde{H}:=\left(m^{2}-\Delta\right)^{-\alpha}\left(1+x^{2}\right)^{-\alpha}
$$

on $L^{2}\left(\mathbb{R}^{d}\right)$, where $\left(1+x^{2}\right)$ is a multiplication operator and $\alpha>d / 4$. Since $\left(1+x^{2}\right)^{-\alpha}$ is square integrable and the same 
is true for the Fourier transform $\left(m^{2}+p^{2}\right)^{-\alpha}$ of $\left(m^{2}-\Delta\right)^{-\alpha}$, the operators $\widetilde{H}$ are of the Hilbert-Schmidt type $\forall \alpha>d / 4$. Like in Section 3, let $\mathscr{H}_{m}^{-}$be the completion of $\mathcal{S}\left(\mathbb{R}^{d}\right)$ with respect to the inner product (3) associated with the operator (41). Taking advantage of the unitary transformation

$$
\left(m^{2}-\Delta\right)^{1 / 4}: L^{2}\left(\mathbb{R}^{d}\right) \longrightarrow \mathscr{H}_{m}^{-}
$$

one can define Hilbert-Schmidt $H$ operator on $\mathscr{H}_{m}^{-}$:

$$
H=\left(m^{2}-\Delta\right)^{1 / 4} \widetilde{H}\left(m^{2}-\Delta\right)^{-1 / 4} \text {. }
$$

Let us finally introduce the following inner product in $\mathcal{S}\left(\mathbb{R}^{d}\right)$ :

$$
\begin{aligned}
& (f, g)_{1}:=\int d^{d} x\left(\left(m^{2}-\Delta\right)^{-1 / 4} H^{-1} f\right) \\
& \cdot\left(\left(m^{2}-\Delta\right)^{-1 / 4} H^{-1} g\right) \\
& =\int d^{d} x\left(\left(1+x^{2}\right)^{\alpha}\left(m^{2}-\Delta\right)^{\beta} f\right) \\
& \cdot\left(\left(1+x^{2}\right)^{\alpha}\left(m^{2}-\Delta\right)^{\beta} g\right),
\end{aligned}
$$

where $\beta=\alpha-1 / 4>(d-1) / 4$. By Theorem 13 , the subspace of those functionals which are continuous with respect to $(,)_{1}$ is a set of measure one. The support of the measure can therefore be written as follows:

$$
\left(m^{2}-\Delta\right)^{\beta}\left(1+x^{2}\right)^{\alpha} L^{2}\left(\mathbb{R}^{d}\right),
$$

in the sense that the distributions which support the measure are such that the application of the operator $\left(1+x^{2}\right)^{-\alpha}\left(m^{2}-\right.$ $\Delta)^{-\beta}$ produces elements of $L^{2}\left(\mathbb{R}^{d}\right)$, for $\alpha>d / 4$ and $\beta>(d-$ 1) $/ 4$.

So, one can say that the Fourier transform of $\left(m^{2}+\right.$ $\left.p^{2}\right)^{-\beta} \widetilde{\phi}(p)$ is locally $L^{2}$, for almost every distribution $\phi(x)$, where $\widetilde{\phi}(p)$ denotes the Fourier transform. Further application of the operator $\left(1+x^{2}\right)^{-\alpha}$ regularizes the behaviour at infinity of typical distributions, producing truly $L^{2}$ elements.

Note that although the value of mass $m$ appears explicitly in the characterization of the support given by (72), the space that one obtains for support of the measure as a consequence of Minlos' theorem is actually the same for all values of the mass. This is a consequence of the fact that the topology defined by the scalar product (71) is independent of the (nonzero) value of the mass. So, the above description of the support of the measure is not sensitive to the value of the mass of the free field.

Nevertheless, as mentioned in Section 6, the measures associated with two distinct values of the mass are in fact singular with respect to each other. Therefore, disjoint supports can be found for distinct masses. Disclosing these crucial differences in the support requires a different type of analysis, namely, one that takes into account the large scale behaviour of typical distributions. We address this question in the next section.

\section{Long Range Behaviour: Distinction of the Supports for Different Values of the Mass}

It is well known that the free field measures $\mu^{m}$ are singular with respect to each other for different values of the mass. In order to distinguish the supports of the measures corresponding to different masses we will now analyze the long range behaviour of typical distributions, which is sensitive to the value of the mass. We adopt here the same method as in [7], with the difference that we are considering now the measure defined by the covariance (41), which is the one appearing in the canonical quantization approach, instead of the corresponding Euclidean path integral measure considered in [7].

In the inverse covariance $C_{m}^{-1}=2\left(m^{2}-\Delta\right)^{1 / 2}$ one can identify a diagonal term, which favours a white noise type of behaviour and a nondiagonal term which imposes correlations between different regions in space, which however decay with distance. So, one can expect that the typical quantum field will present strong correlations for small distances and will approach white noise behaviour at large distances. The scale distance is clearly marked by the value of the mass; that is, $\mathrm{m}^{-1}$ can be interpreted as a correlation lenghth. The expected behaviour at scales much larger than $\mathrm{m}^{-1}$ is therefore that of a white noise type of measure with covariance $\sigma=(2 m)^{-1}$.

In order to obtain our formal result, let us consider the measurable functions $F_{B_{j}}: \mathcal{S}^{\prime}\left(\mathbb{R}^{d}\right) \rightarrow \mathbb{C}$ given by

$$
F_{B_{j}}: \phi \longmapsto F_{B_{j}}(\phi) \equiv \phi\left(f_{j}\right)=\frac{1}{L^{d}} \int_{B_{j}} \phi(x) d^{d} x,
$$

where $\left\{B_{j}\right\}_{j=1}^{\infty}$ is a family of mutually disjoint hypercubes in $\mathbb{R}^{d}$, of edge length $L$, and $f_{j}$ denotes the characteristic function of the hypercube $B_{j}$ multiplied by $1 / L^{d}$. We will consider the family of hypercubes $\left\{B_{j}\right\}_{j=1}^{\infty}$ centered at points $x^{j}=\left(x_{1}^{j}, x_{2}^{j}, \ldots, x_{d}^{j}\right)=\left(j^{2} / m, 0, \ldots, 0\right)$ with faces parallel to the coordinate planes.

The push-forward of the free field measure $\mu^{m}$ with respect to the map

$$
\begin{aligned}
\mathcal{S}^{\prime}\left(\mathbb{R}^{d}\right) & \longrightarrow \mathbb{R}^{\mathbb{N}} \\
\phi & \longmapsto\left\{\phi\left(f_{j}\right)\right\}
\end{aligned}
$$

is the Gaussian measure $\nu_{m}$ in $\mathbb{R}^{\mathbb{N}}$ with covariance matrix $\mathscr{M}_{m}$ given by

$$
\left(\mathscr{M}_{m}\right)_{j l}=\left\langle f_{j}, C_{m} f_{l}\right\rangle .
$$

The matrix elements can be easily found by Fourier transform. We get

$$
\begin{aligned}
\left(\mathscr{M}_{m}\right)_{j l}= & \frac{1}{2}\left(\frac{2}{\pi}\right)^{d} \frac{1}{L^{2 d}} \int_{\mathbb{R}^{d}} d^{d} k e^{i\left(k_{1} / m\right)\left(j^{2}-l^{2}\right)} \\
& \cdot \frac{1}{\left(k^{2}+m^{2}\right)^{1 / 2}} \prod_{n=1}^{d} \frac{\sin ^{2}\left(k_{n} L / 2\right)}{k_{n}^{2}} .
\end{aligned}
$$


The Fourier transform in (76) is well defined, since

$$
\frac{1}{\left(k^{2}+m^{2}\right)^{1 / 2}} \prod_{n=1}^{d} \frac{\sin ^{2}\left(k_{n} L / 2\right)}{k_{n}^{2}}
$$

is absolutely integrable in $\mathbb{R}^{d}$, which also shows that $f_{j} \in \mathscr{H}_{m}^{-}$, $\forall j$, proving therefore that the map (74) is well defined (see Section 2.1). As expected, due to the invariance of the measure with respect to spatial translations, the diagonal elements of the matrix $\mathscr{M}_{m}$ are all equal. Let us denote them by $\lambda_{m}^{L}$ :

$$
\begin{aligned}
\lambda_{m}^{L} & :=\left(\mathscr{M}_{m}\right)_{j j}=\frac{1}{2}\left(\frac{2}{\pi}\right)^{d} \frac{1}{L^{2 d}} \int_{\mathbb{R}^{d}} d^{d} k \frac{1}{\left(k^{2}+m^{2}\right)^{1 / 2}} \\
& \cdot \prod_{n=1}^{d} \frac{\sin ^{2}\left(k_{n} L / 2\right)}{k_{n}^{2}}
\end{aligned}
$$

and let $\mu_{\lambda_{m}^{L}}$ be the Gaussian measure in $\mathbb{R}^{\mathbb{N}}$ of diagonal covariance matrix $\lambda_{m}^{L} \mathbf{1}$ (where $\mathbf{1}$ is the diagonal matrix in $\mathbb{R}^{\mathbb{N}}$ ).

Lemma 14. The measures $\nu_{m}$ and $\mu_{\lambda_{m}^{L}}$ are mutually absolutely continuous; that is, they have the same zero measure sets.

To prove the lemma we will rely on Theorem I.23 of [12] (see also Theorem 10.1 of [14]), which gives necessary and sufficient conditions for two covariances to give rise to mutually absolutely continuous Gaussian measures. In the present case, since the covariance of $\mu_{\lambda_{m}^{L}}$ is proportional to the identity, it is sufficient to show that (i) $\mathscr{M}_{m}$ is bounded and positive with bounded inverse in $\ell^{2}$ and (ii) $T:=\mathscr{M}_{m}-\lambda_{m}^{L} \mathbf{1}$ is Hilbert-Schmidt in $\ell^{2}$. The fact that $\mathscr{M}_{m}$ is positive and injective follows from the fact that $\mathscr{M}_{m}$ is the restriction of $C_{m}$ to the linearly independent system $\left\{f_{j}\right\}_{j \in \mathbb{N}}$. Let us admit for a moment that (ii) is proved. It is then clear that $\mathscr{M}_{m}$ is bounded, since it is the sum of two bounded operators. Let us suppose that $\mathscr{M}_{m}$ does not have a bounded inverse. Then, by definition, $-\lambda_{m}^{L}$ belongs to the spectrum of $T$. But $T$ is compact and therefore the (nonzero) points of the spectrum are proper values, which contradicts the injectivity of $\mathscr{M}_{m}$. Thus, it remains only to show that $T:=\mathscr{M}_{m}-\lambda_{m}^{L} \mathbf{1}$ is Hilbert-Schmidt. The matrix elements of $T$ are $T_{j j}=0$ and $T_{j l}=\left(\mathscr{M}_{m}\right)_{j l}$ for $j \neq l$. One can conclude from (76) that the nondiagonal elements $\left(\mathscr{M}_{m}\right)_{j l}$ are the values at points $j^{2}-l^{2}$ of the Fourier transform of a real function $f$. In fact, taking into account the change of variable $p_{n}=k_{n} / m$, we get

$$
\left(\mathscr{M}_{m}\right)_{j l}=\int_{\mathbb{R}} d p_{1} e^{i p_{1}\left(j^{2}-l^{2}\right)} f\left(p_{1}\right)
$$

where

$$
\begin{aligned}
f\left(p_{1}\right):= & \frac{1}{2}\left(\frac{2}{\pi}\right)^{d} \frac{1}{m^{d+1} L^{2 d}} \frac{\sin ^{2}\left(m L p_{1} / 2\right)}{p_{1}^{2}} \\
& \cdot \int_{\mathbb{R}^{d-1}} d^{d-1} p \frac{1}{\left(1+p_{1}^{2}+\sum_{2}^{d} p_{n}^{2}\right)^{1 / 2}} \\
& \cdot \prod_{2}^{d} \frac{\sin ^{2}\left(m L p_{n} / 2\right)}{p_{n}^{2}} .
\end{aligned}
$$

The above function $f$ is clearly differentiable and its derivative $f^{\prime}$ belongs to $L^{1}(\mathbb{R})$. We therefore obtain for the Fourier transform $\tilde{f}$ :

$$
\tilde{f}\left(p_{1}\right)=\frac{\widetilde{f^{\prime}}\left(p_{1}\right)}{i p_{1}}
$$

with $\widetilde{f^{\prime}}$ being continuous and bounded and approaching zero at infinity. One can therefore find $A, 0<A<\infty$, such that

$$
\left|\tilde{f}\left(p_{1}\right)\right| \leq \frac{A}{\left|p_{1}\right|} .
$$

Passing to the matrix elements $\left(\mathscr{M}_{m}\right)_{j l}$ we get

$$
\left|\left(\mathscr{M}_{m}\right)_{j l}\right|^{2} \leq \frac{A}{\left(j^{2}-l^{2}\right)^{2}}, \quad \text { for } j \neq l .
$$

Using the standard $\ell^{2}$ basis $e_{k}=\left(\delta_{k n}\right)$ we finally obtain

$$
\sum_{l}\left\langle T e_{l}, T e_{l}\right\rangle=\sum_{j, l}\left|T_{j l}\right|^{2} \leq A \sum_{j \neq l} \frac{1}{\left(j^{2}-l^{2}\right)^{2}}<\infty,
$$

showing that $T$ is Hilbert-Schmidt.

Proposition 15. Let $\mu_{\rho}$ be the Gaussian measure in $\mathbb{R}^{\mathbb{N}}$ of diagonal covariance $\rho \mathbf{1}$, with $\rho>0$. Consider the measurable subsets of $\mathbb{R}^{\mathbb{N}}$ defined by

$$
\begin{aligned}
Z_{\rho}^{\epsilon} & :=\left\{x \mid \exists N_{x} \in \mathbb{N} \text { such that }\left|x_{n}\right|\right. \\
& \left.<\sqrt{2(1+\epsilon) \rho \ln n}, \text { for } n \geq N_{x}\right\},
\end{aligned}
$$

where $\epsilon \geq 0$. Then the sets $Z_{\rho}^{\epsilon}$ have $\mu_{\rho}$ measure equal to one for all $\epsilon>0$. The set $Z_{\rho}^{0}$ has $\mu_{\rho}$ measure equal to zero.

This proposition can be proven as follows. Consider the measurable sets $Z_{\rho}^{\epsilon}(N), N \in \mathbb{N}$, defined by

$$
Z_{\rho}^{\epsilon}(N):=\{x|| x \mid<\sqrt{2(1+\epsilon) \rho \ln n}, \text { for } n \geq N\} .
$$

Since the measure $\mu_{\rho}$ is in fact a product measure of identical Gaussian measures in $\mathbb{R}$, it is not difficult to see that $\mu_{\rho}$ measure of this sets is

$$
\Lambda_{N}^{\epsilon}:=\mu_{\rho}\left(Z_{\rho}^{\epsilon}(N)\right)=\prod_{n=N}^{\infty} \operatorname{Erf}(\sqrt{(1+\epsilon) \ln n}),
$$


where $\operatorname{Erf}(x)=1 / \sqrt{\pi} \int_{-x}^{x} e^{-\xi^{2}} d \xi$ is the error function. Given that

$$
\begin{array}{r}
Z_{\rho}^{\epsilon}(N) \subset Z_{\rho}^{\epsilon}(N+1), \\
Z_{\rho}^{\epsilon}=\bigcup_{N \in \mathbb{N}} Z_{\rho}^{\epsilon}(N),
\end{array}
$$

it follows from $\sigma$-additivity of the measure that

$$
\mu_{\rho}\left(Z_{\rho}^{\epsilon}\right)=\lim _{N \rightarrow \infty} \Lambda_{N}^{\epsilon}
$$

Since, in particular, the infinite product $\Lambda_{2}^{\epsilon}$ exists $\left(0 \leq \Lambda_{2}^{\epsilon} \leq\right.$ 1 ), we have that $\lim _{N \rightarrow \infty} \Lambda_{N}^{\epsilon}=1$ or $\Lambda_{2}^{\epsilon}=0$. The condition $\Lambda_{2}^{\epsilon}=0$ is equivalent to $\Lambda_{N}^{\epsilon}=0, \forall N>1$, given that $\operatorname{Erf}(\sqrt{(1+\epsilon) \ln n})>0, \forall n>1$. So, $\mu_{\rho}\left(Z_{\rho}^{\epsilon}\right)$ is equal to zero if $\Lambda_{2}^{\epsilon}=0$ and equal to one otherwise. Passing to logarithms, it remains to consider the convergence of the infinite sum

$$
\sum_{n=2}^{\infty} \ln \left(\operatorname{Erf}\left(\sqrt{\ln n^{1+\epsilon}}\right)\right) .
$$

Replacing the general term $\ln \left(\operatorname{Erf}\left(\sqrt{\ln n^{1+\epsilon}}\right)\right)$ in (90) by its asymptotic limit, namely, $-1 /\left(n^{1+\epsilon} \sqrt{(1+\epsilon) \ln n}\right)$, we conclude that the sum (90) diverges for $\epsilon=0$ and is convergent $\forall \epsilon>0$.

Finally, by combining Lemma 14 with Proposition 15, we immediately obtain the following result concerning the support of the free field measures $\mu^{m}$.

Theorem 16. The sets

$$
\begin{aligned}
Y_{m}^{\epsilon} & :=\left\{\phi \in \mathcal{S}^{\prime}\left(\mathbb{R}^{d}\right) \mid \exists N_{\phi} \in \mathbb{N} \text { such that }\left|\phi\left(f_{n}\right)\right|\right. \\
& \left.<\sqrt{2(1+\epsilon) \lambda_{m}^{L} \ln n}, \text { for } n \geq N_{\phi}\right\}
\end{aligned}
$$

have $\mu^{m}$ measure one for any $\epsilon>0$ and measure zero for $\epsilon=0$. For $m^{\prime}<m$ there exists $\epsilon\left(m^{\prime}\right)>0$ such that $\mu^{m^{\prime}}\left(Y_{m}^{\epsilon\left(m^{\prime}\right)}\right)=0$.

The second statement of the theorem, which in particular allows the construction of disjoint supports for $m \neq m^{\prime}$, follows from the strict monotony of $\lambda_{m}^{L}$ having a function of $m$.

\section{Conclusions}

Measures in infinite dimensional spaces, both linear and nonlinear, are of major importance in quantum theory. They appear already in standard Quantum Mechanics, in the path integral formulation, but are even more relevant in the quantum theory of fields, both from the Euclidean path integral and from the canonical quantization perspectives (see, e.g., [6]), including the quantization of gravity (see $[17,18])$. These measures are typically defined on extensions of the spaces associated with the classical field theories in question. In the case of scalar fields one finds measures in the space $S^{\prime}\left(\mathbb{R}^{d}\right)$ of tempered distributions, dual of the Schwartz space.
In all known cases the distributional extensions are crucial, given that, for measures of interest, the classical nondistributional configurations turn out to be (subsets of) sets of measure zero.

The construction of measures corresponding to field theories with interactions turned out to be a problem of major complexity, in particular for spacetime dimensions above $1+1$ (see, nevertheless, $[2,3,6,11,12]$ ). The quantum theory of the free scalar field (in arbitrary $d+1$ dimensions), however, is well established and has been extensively studied under several viewpoints. The simplification resides, of course, in the linearity of the dynamics: the free field Hamiltonian is quadratic, allowing a quantization of the model by means of well defined Gaussian measures. In the canonical formalism, the free field in $d+1$ dimensions is quantized using a Gaussian measure in $\mathcal{S}^{\prime}\left(\mathbb{R}^{d}\right)$ which is effectively determined by the classical Hamiltonian. The measure is invariant under the action of the Euclidean group in $\mathbb{R}^{d}$ and provides simultaneously a representation of the Weyl relations and of the action of the Poincaré group. In particular, one obtains a natural quantization of the dynamics $[1,2]$.

It is well known that the representations associated with free fields with different values of the mass are not unitarily equivalent. This nonequivalence reflects the fact that the measures corresponding to distinct masses are supported in different-in fact disjoint-sets of $\mathcal{S}^{\prime}\left(\mathbb{R}^{d}\right)$. In Section 9 we studied the differences between the supports of the measures for distinct masses: we singled out properties which are specific of the "typical (quantum) field" for a given value of the mass. We have therefore looked for a characterization as refined as possible of the set of elements of $\mathcal{S}^{\prime}\left(\mathbb{R}^{d}\right)$ that effectively contributes to a given free field measure. Since the support of the measure replaces the classical configuration space in the representations of the kinematical algebra, this analysis corresponds to a characterization of the "quantum configuration space" for a given value of the free field mass. For this purpose, we considered a sequence of stochastic variables that test the behaviour of the field for large distances.

Finally, let us mention that the study of the support of the free field measures is important not only for a good understanding of these models but also from the broader perspective of the construction of quantum theories with interactions. In the usual procedure, the introduction of interactions in a free model is constrained by the properties of the "typical free fields": the local (distributional) behaviour leads to the well known ultraviolet divergences, whereas the long range behaviour is related to infrared divergences $[2,3$, $6,9,11,12]$.

\section{Conflict of Interests}

The author declares that there is no conflict of interests regarding the publication of this paper.

\section{References}

[1] J. C. Baez, I. E. Segal, and Z.-F. Zhou, Introduction to Algebraic and Constructive Quantum Field Theory, Princeton University Press, 1992. 
[2] M. Reed and B. Simon, Methods of Modern Mathematical Physics, vol. II, Academic Press, 1975.

[3] M. Reed and B. Simon, Methods of Modern Mathematical Physics, vol. III, Academic Press, 1979.

[4] R. Haag, Local Quantum Physics, Texts and Monographs in Physics, Springer, Berlin, Germany, 1992.

[5] I. M. Gel'fand and N. Y. Vilenkin, Generalized Functions, vol. 4, Academic Press, New York, NY, USA, 1964.

[6] J. Glimm and A. Jaffe, Quantum Physics, Springer, 1987.

[7] J. M. Mourão, T. Thiemann, and J. M. Velhinho, "Physical properties of quantum field theory measures," Journal of Mathematical Physics, vol. 40, no. 5, pp. 2337-2353, 1999.

[8] J. M. Velhinho, "Local properties of measures in quantum field theory and cosmology," Symmetry, Integrability and Geometry: Methods and Applications, vol. 11, article 006, 2015.

[9] M. Reed and L. Rosen, "Support properties of the free measure for Boson fields," Communications in Mathematical Physics, vol. 36, pp. 123-132, 1974.

[10] P. Colella and O. E. Lanford, "Appendix: sample field behavior for the free Markov random field," in Constructive Quantum Field Theory, The 1973 Ettore Majorana School, G. Velo and A. Wightman, Eds., vol. 25 of Lecture Notes in Physics, pp. 44-70, Springer, Berlin, Germany, 1973.

[11] V. Rivasseau, From Perturbative to Constructive Renormalization, Princeton University Press, 1991.

[12] B. Simon, The $P(\emptyset)_{2}$ Euclidean (Quantum) Field Theory, Princeton University Press, 1974.

[13] W. Rudin, Functional Analysis, McGraw-Hill, 1991.

[14] Y. Yamasaki, Measures on Infinite Dimensional Spaces, World Scientific, 1985.

[15] M. Reed and B. Simon, Methods of Modern Mathematical Physics, vol. 1, Academic Press, 1980.

[16] Y. G. Sinai, Topics in Ergodic Theory, Princeton University Press, 1994.

[17] A. Ashtekar and J. Lewandowski, "Background independent quantum gravity: a status report," Classical and Quantum Gravity, vol. 21, no. 15, pp. R53-R152, 2004.

[18] T. Thiemann, Modern Canonical Quantum General Relativity, Cambridge University Press, 2007. 


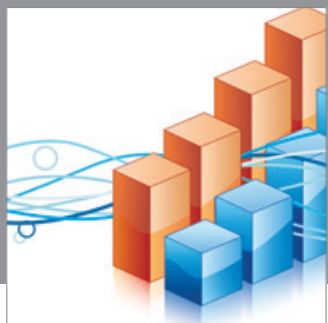

Advances in

Operations Research

mansans

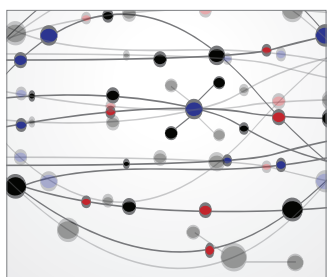

The Scientific World Journal
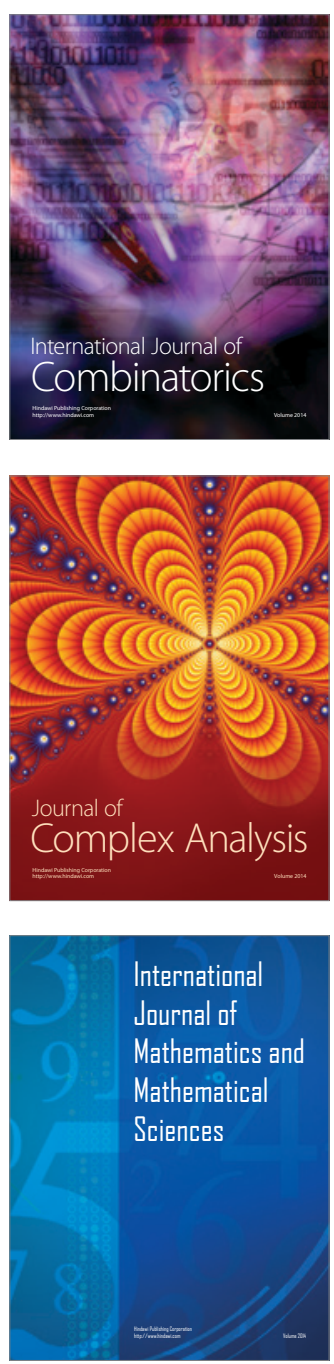
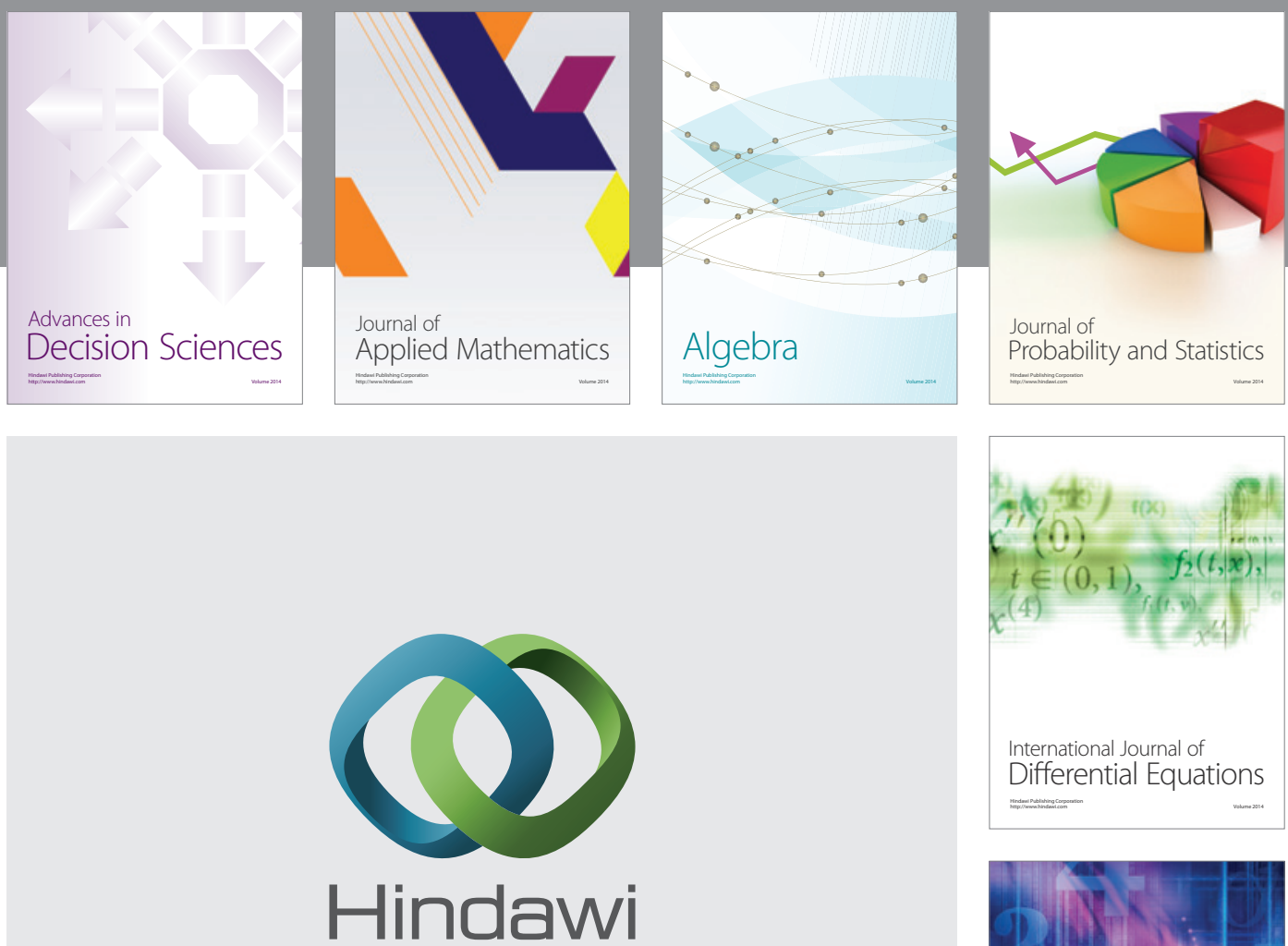

Submit your manuscripts at http://www.hindawi.com
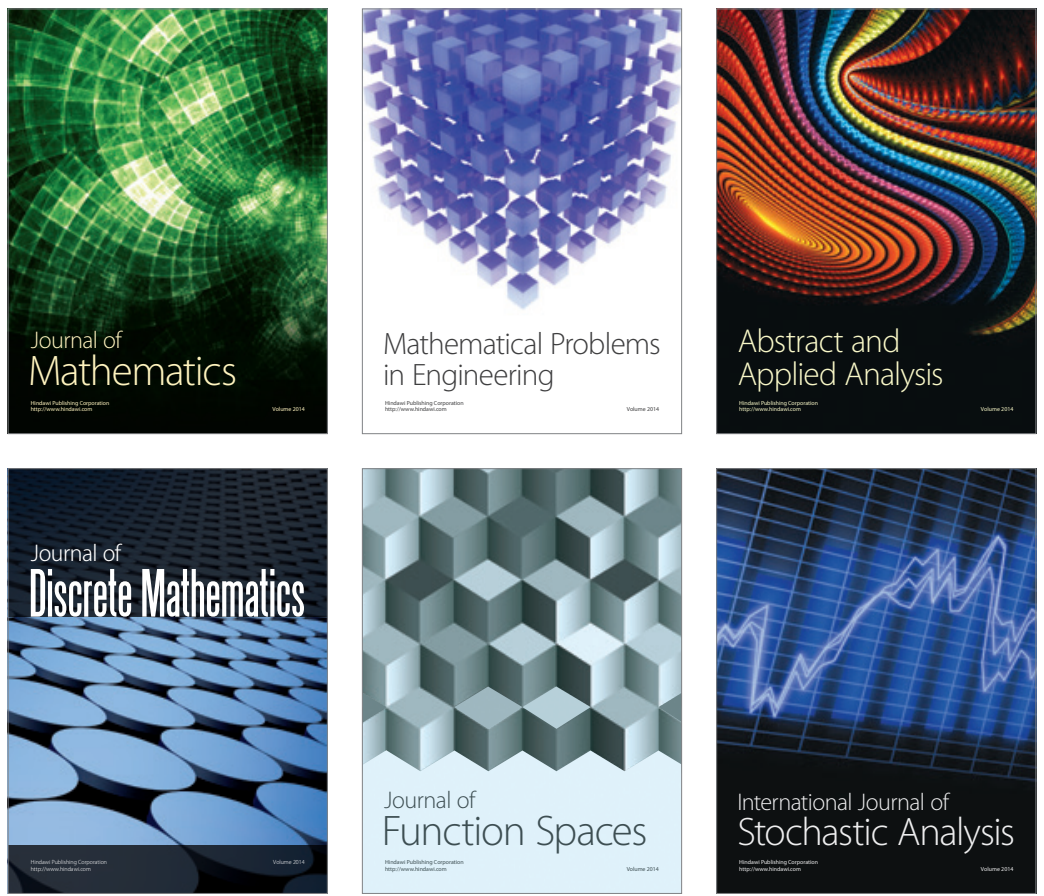

Journal of

Function Spaces

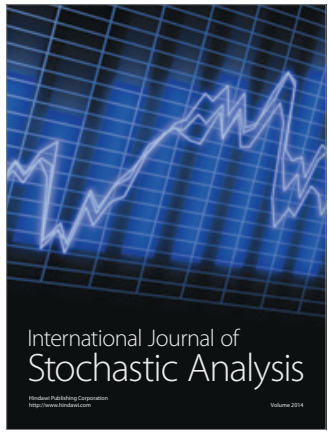

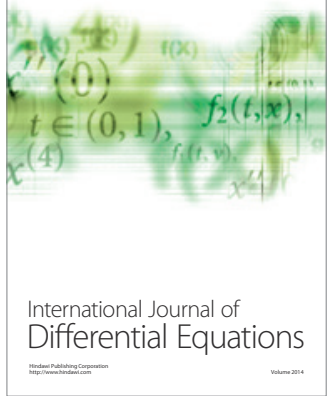
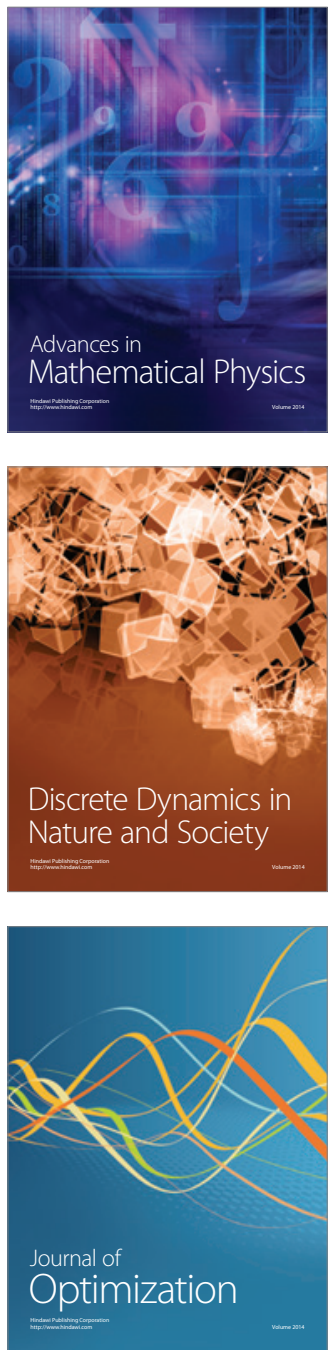